\title{
Le coût du SIDA
}

$\mathbf{L}$ ongtemps réservés sur l'étude des coûts de la maladie, les économistes ont dû changer d'attitude pour le SIDA. Le nombre de travaux consacrés à ce sujet est maintenant impressionnant et mérite d'être expliqué, puisqu'il illustre un changement déterminant en économie de la santé. Les deux raisons principales des réticences des économistes tenaient, d'une part, à l'imperfection des méthodes d'imputation des coûts et, d'autre part, à l'ambiguïté des objectifs de telles études.

Sur le premier point, l'imputation des frais de personnel ou du matériel utilisé permet de larges variations, entre ceux qui comptabilisent tout le personnel impliqué (y compris une fraction du personnel administratif de l'hôpital), et ceux qui calculent au plus juste, en ne retenant que les consommations directement liées au traitement de la maladie étudiée. Un autre élément de grande diversité résulte dans la valorisation des coûts indirects (perte de revenus, absentéisme...) ou intangibles (prix de la douleur, de l'inquiétude, de l'attente...).

Il est vrai que lorsque le plein emploi n'est pas garanti, le fait de considérer la maladie comme un facteur de privation de ressources est une approximation, que sa correction expose à des interprétations parfois hasardeuses...

Par ailleurs, les économistes se méfient des objectifs poursuivis, parfois de manière peu explicite, par les études de coûts des maladies. Souvent, il s'agit, pour certains spécia- listes d'une discipline, de dénoncer soit l'insuffisance de crédits consacrés à leur spécialité, soit au contraire d'insister sur son poids trop lourd, afin de sensibiliser les pouvoirs publics sur des mesures de prévention ou de recherche qui pourraient les atténuer.

Les études sur le SIDA ne sont pas exemptes de ces reproches, mais la justification de leur poursuite réside dans les caractéristiques propres de cette maladie.

Le SIDA est une maladie nouvellement identifiée, nécessitant des moyens nouveaux de diagnostic et de prise en charge qui s'ajoutent aux dépenses de soins classiques. Il est donc important de prévoir quelles sommes devront être allouées spécifiqueinent pour ce poste nouveau, d'autant plus qu'aucune substitution ne peut s'opérer à partir des soins pour les autres maladies.

Le SIDA est la première maladie pour laquelle un "dossier médicoéconomique de l'immuno-déficience humaine (DMI) a été mis en place, en France comme dans de nombreux pays, pour réunir les éléments perti-

\begin{tabular}{|lc|}
\hline Structures & $\begin{array}{l}\text { Présents en moyenne } \\
\text { (par jour) }\end{array}$ \\
\hline Hospitalisation complète & 1550 \\
Hôpital de jour & 500 \\
Consultation & 350 \\
Hospitalisation à domicile & 250 \\
Moyen séjour & 150 \\
Total & $\mathbf{3} 800$ \\
\hline
\end{tabular}

nents du dossier médical des malades et les transférer sur un support anonyme, informatisé et centralisé. Par ailleurs, une enquête nationale, en France, est organisée chaque semestre, "un jour donné ", permettant d'apprécier la structure et l'évolution de la fréquentation hospitalière des malades dans l'ensemble des hôpitaux publics ou privés. Les données apportées par l'exploitation du DMI et celles de l'enquête semestrielles sont indépendantes, et la validation mutuclle des informations est donc possible.

En 1990, les hôpitaux français ont accueilli chaque jour 3800 patients atteints par le HIV, dont $55 \%$ étaient sidéens. Le Tableau ci-dessous résume la fréquentation quotidienne moyenne dans chaque structure.

A partir de ces données, on peut évaluer le montant total des dépenses consacrées à la pathologie HIV à l'intérieur du système hospitalier français : elle s'élève à 2,2 milliards de francs en 1990, en croissance de $22 \%$ par rapport à 1989. Cette 
somme représcnte environ $1 \%$ de la dépense totalc hospitalière (210 milliards en 1990).

Dans cet effort financier, il est intéressant d'individualiser certains postes : les médicaments et examens de laboratoire représentent $33 \%$ de la dépense, l'essentiel (64\%) étant représenté par le personnel, très sollicité par les soins lourds et une présence humaine très personnalisée (évaluée très précisément à 6 heures/jour de soins infirmiers en hospitalisation complète). La durée moyenne de séjour d'un malade pour un épisode aigu en rapport avec le HIV cst de 17 jours, le nombre moyen de séjours étant de 2 à 3 par an. Malgré l'apparition croissantc de formes plus graves de la maladie (formes ncurologiques et lymphomes), les séjours hospitalicrs ont plutôt tendance à raccourcir, de sorte que le coût annuel moyen du traitement d'un malade reste stable malgré les nouveaux traitements (150 000 F à $200000 \mathrm{~F}$ ).

Les soins en médecine ambulatoire (hors AZT, distribué par les pharmacies hospitalières publiques), qui intéressent près de 30000 personnes, coûtent pour leur part $150 \mathrm{MF}$.

Parmi les médicaments, le seul AZT est devenu lc principal poste de consommation des pharmacics hospitalière (devant l'héparine), et coûte environ $150 \mathrm{MF} / \mathrm{an}$ (soit autant que tous les soins ambulatoires). Ce poids économique est tel qu'une variation dans la posologie de l'ordre de $100 \mathrm{mg}$ a une incidence sur la dépense d'environ $25 \mathrm{MF}$.

L'effort de prévention contre le SIDA, coordonné par l'Agence Françaisc de Lutte contre le SIDA, a mobilisé en 1990 un budget d'cnvi- ron 78 MF, réparti à peu près également entre des actions de communication et des "actions de terrain". Les sommes consacrées à la recherche s'élèvent à $440 \mathrm{MF}$ pour les années 1989-1990, dont $54 \%$ dans lc secteur public, $17 \%$ dans l'industric, $20 \%$ dans les associations et fondations, et $6 \%$ pour les bourses.

Aux États-Unis, une estimation pour 1991 avait été réalisée en 1987 par les autorités fédérales, situant à 66,5 milliards de dollars le coût total annuel de la maladie, dont 10,9 milliards de coût direct des traitements, et 55,6 milliards dc coûts indirects, principalement représentés par les pertes de revenus occasionnées par les décès prématurés des malades 